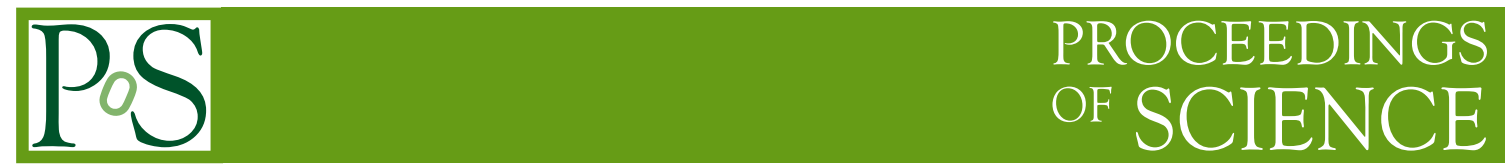

\title{
Astrophysics with the AMS-02 experiment
}

\author{
Rui Pereira* \\ on behalf of the AMS collaboration \\ LIP Lisbon \\ Lisbon, Portugal \\ E-mail: pereira@lip.pt
}

The Alpha Magnetic Spectrometer (AMS), whose final version AMS-02 is to be installed on the International Space Station (ISS) for at least 3 years, is a detector designed to measure charged cosmic ray spectra with energies up to the $\mathrm{TeV}$ region and with high energy photon detection capability up to a few hundred $\mathrm{GeV}$, using state-of-the-art particle identification techniques. Following the successful flight of the detector prototype (AMS-01) aboard the space shuttle, AMS-02 is expected to provide a significant improvement on the current knowledge of the elemental and isotopic composition of hadronic cosmic rays due to its long exposure time (minimum of 3 years) and large acceptance $\left(0.5 \mathrm{~m}^{2} \mathrm{sr}\right)$ which will enable it to collect a total statistics of more than $10^{10}$ nuclei. Detector capabilities for charge, velocity and mass identification, estimated from ion beam tests and detailed Monte Carlo simulations, are presented. Relevant issues in cosmic ray astrophysics addressed by AMS-02, including the test of cosmic ray propagation models, galactic confinement times and the influence of solar cycles on the local cosmic ray flux, are briefly discussed.

International Europhysics Conference on High Energy Physics

July 21st - 27th 2005

Lisboa, Portugal

${ }^{*}$ Speaker. 


\section{The AMS experiment}

Alpha Magnetic Spectrometer (AMS)[1] is an experiment designed to study the cosmic ray flux by direct detection of particles above the Earth's atmosphere. The the final detector (AMS-02) will be ready for launch by the end of 2007 and installed on the International Space Station (ISS) for more than 3 years. A preliminary version of the detector (AMS-01) was successfully flown aboard the US space shuttle Discovery in June 1998.

On the ISS, orbiting at an average altitude of $400 \mathrm{~km}$, AMS-02 will collect an extremely large number of cosmic ray particles. Its main goals are (i) a detailed study of cosmic ray composition and energy spectrum through the collection of an unprecedented volume of data, (ii) a search for heavy antinuclei $(Z \geq 2)$ which if discovered would signal the existence of cosmological antimatter (the detection of anti-carbon $(Z=6)$ would be evidence for the existence of anti-stars), and (iii) a search for dark matter constituents by examining possible signatures of their presence in the cosmic ray spectrum. AMS-02 is equipped with a superconducting magnet cooled by superfluid helium. The spectrometer is composed of several subdetectors: a Transition Radiation Detector (TRD), a Time-of-Flight (TOF) detector, a Silicon Tracker, Anticoincidence Counters (ACC), a Ring Imaging Čerenkov (RICH) detector with a dual radiator (silica aerogel and sodium fluoride) and an Electromagnetic Calorimeter (ECAL). Fig. 1 shows a schematic view of the full AMS-02 detector.

\section{Detector requirements}

To fulfill its astrophysics goals, AMS-02 must be able to detect nuclei and identify their charge in a large range of $Z$. Precise velocity and rigidity measurements are also required, namely to discriminate between different isotopes of the same element.

Cosmic ray propagation models may be tested by comparing abundances of primary and secondary species at different energies. Primary cosmic rays are those produced at the original sources, while secondaries are created through the interaction of primary cosmic rays with the interstellar medium. Abundance ratios such as the one between boron $(Z=5$, secondary) and carbon $(Z=6$, primary), shown in fig. 2, depend on the details of cosmic ray propagation.

Data on confinement times may be inferred from the comparison between stable and unstable nuclei with similar origin. This is the case of beryllium isotopes ${ }^{9} \mathrm{Be}$ (stable) and ${ }^{10} \mathrm{Be}$ (radioactive, $t_{1 / 2}=1.6 \mathrm{Myr}$ ), which are both secondary species.

\section{Measurement of particle properties}

In AMS-02 the evaluation of particle charge is performed using data from the Silicon Tracker, TOF and RICH detectors. In the Tracker and TOF, charge is given by direct sampling of local energy deposition along the particle's path: $\Delta E \propto Z^{2}$. In the case of the RICH detector the energy deposition is also proportional to $Z^{2}$ but it is spread over a large region (Čerenkov ring). The charge sign is determined from the bending of the trajectory in the Silicon Tracker, combined with albedo rejection capabilities from the TOF and RICH detectors. The expected charge resolution $\Delta Z$ from redundant measurements in the TOF, Tracker and RICH is $\Delta Z \lesssim 0.25$ charge units up to the iron region $(Z=26)$. 


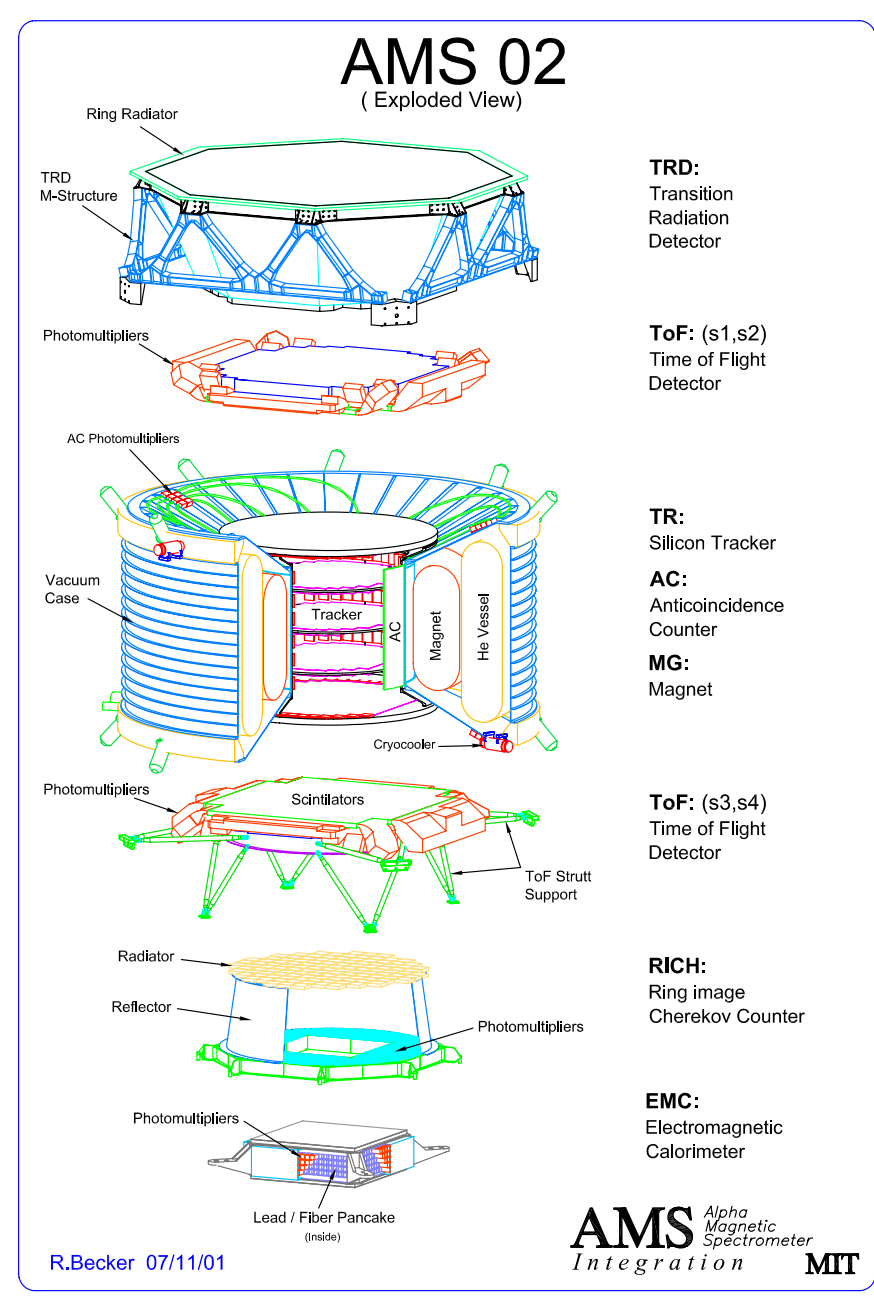

Figure 1: Exploded view of the AMS-02 detector

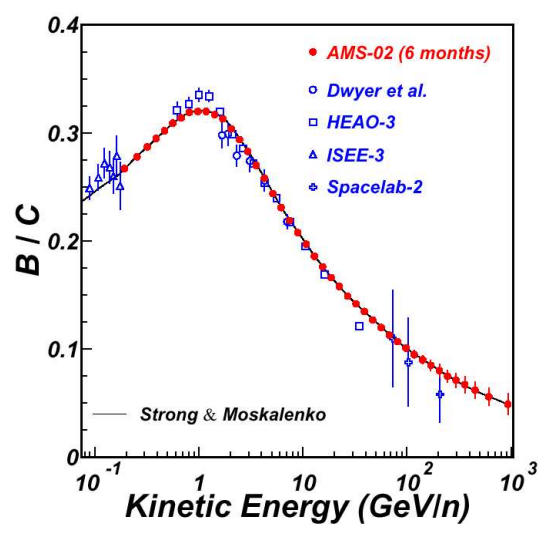

Figure 2: AMS-02 expected performance for elemental ratio $\mathrm{B} / \mathrm{C}$ after 6 months of data taking compared with existing results.

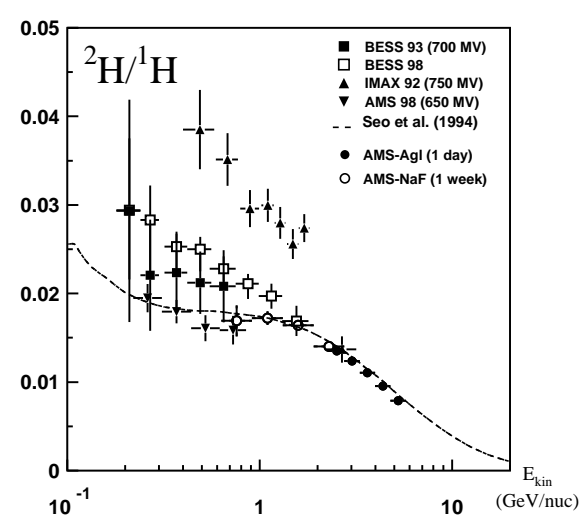

Figure 3: Reconstruction of simulated isotopic ratio D/p in AMS-02 compared with results from previous experiments.

Particle velocity may be obtained from the TOF and RICH detectors. In the Time of Flight detector, velocity is calculated from the crossing time $t$ between scintillator planes. In AMS-02 the expected accuracy for protons is $\Delta t \sim 130 \mathrm{ps}$, leading to $\Delta \beta / \beta \sim 4 \%$. In the RICH detector, velocity is calculated from the aperture of the Čerenkov cone $\left(\theta_{c}\right): \cos \theta_{c}=\frac{1}{\beta n}$ where $n$ is the radiator's refractive index. The typical accuracy in AMS- 02 is $\Delta \beta / \beta \sim 10^{-3}$ for $Z=1$, and $\Delta \beta / \beta \sim 10^{-4}$ for $Z>10$. However, the RICH measurement is only available if the particle's kinetic energy $E_{\text {kin }}$ exceeds the Čerenkov threshold for the radiator crossed: $E_{k i n}>0.5 \mathrm{GeV} /$ nucleon for sodium fluoride $(\mathrm{NaF})\left(\sim 10 \%\right.$ of events), and $E_{k i n}>2.1 \mathrm{GeV} /$ nucleon for silica aerogel (the remaining $\left.\sim 90 \%\right)$.

Additionally, the Transition Radiation Detector combined with the Electromagnetic Calorimeter provides $e / p$ separation up to $\sim 1 \mathrm{TeV}$ with a discrimination factor of $\sim 10^{5}$.

Mass identification in AMS-02 relies on precise velocity and rigidity measurements, where the rigidity $R$, defined as $R=p / Z$, is provided by the sampling of the particle's trajectory given by the Silicon Tracker. For protons with $E_{k i n} \sim 1-10 \mathrm{GeV}$, the accuracy is $\Delta R / R \sim 2 \%$. The final mass estimate is given by $m=\frac{R Z}{\gamma v}$ where $\gamma$ is the Lorentz factor. The expected mass resolution is of the order of $2-3 \%$ for light nuclei $(Z \lesssim 5)$ with $E_{k i n} \sim 5 \mathrm{GeV} /$ nucleon. The effect of the mass resolution 
in the isotopic separation depends also on the isotopic ratio. Separation of isotopes will be possible up to high kinetic energies: at least $6 \mathrm{GeV} /$ nucleon for hydrogen (fig. 3), where the limit comes from the large proton background $\left(D / p \sim 10^{-2}\right)$, and $8 \mathrm{GeV} /$ nucleon in the case of elements such as $\mathrm{He}$ and $\mathrm{Be}$.

\section{AMS-02 prospects}

Fig. 2 shows the expected performance in the determination of the elemental ratio B/C after 6 months of data taking. AMS-02 results will represent a major improvement with respect to existing data, giving information on abundances of these (and other) elements up to energies of $\sim 1 \mathrm{TeV} /$ nucleon.

Fig. 3 shows the results for the isotopic ratio D/p as a function of energy in two simulations of hydrogen events[2], one consisting only of particles crossing the NaF radiator of the RICH detector and the other corresponding to particles crossing the aerogel radiator. Events were simulated according to the reacceleration model of reference [3] represented by the dashed line. The total statistics was $\sim 1.5 \times 10^{7}$ events in each case, which corresponds to $\sim 1$ week of data in the case of $\mathrm{NaF}$ and $\sim 1$ day in the case of aerogel. In both cases, the results obtained show a large improvement with respect to existing data from previous experiments.

It should be noted that solar cycles have a significant effect on the ratio D/p (effects on heavier elements are much smaller). The long operating period of AMS-02 will allow to collect data over a significant fraction of a solar cycle (11 years on average between successive maxima), and its high acceptance will provide enough statistics to obtain ratio samplings every few days.

\section{Conclusions}

Data from AMS-02 will provide a major improvement with respect to existing results for the hadronic cosmic ray spectrum. A total statistics of more than $10^{10}$ events will be collected during its operation. Detector capabilities include charge separation up to $Z \sim 30$, velocity reconstruction with $\Delta \beta / \beta \sim 10^{-3}$ for $Z=1$ and $\Delta \beta / \beta \sim 10^{-4}$ for $Z \sim 10-20$, and isotopic separation of light elements. Results of AMS-02 will address key issues in cosmic ray astrophysics, namely, propagation of cosmic rays in the Galaxy, confinement times, and the effect of solar cycles on the flux in Earth's vicinity.

\section{References}

[1] S. P. Ahlen et al., Nucl. Instrum. Methods A 350, 34 (1994).

V. M. Balebanov et al., AMS proposal to DOE, approved April 1995.

[2] R. Pereira et al., Isotope separation with the RICH detector of the AMS experiment, in Proceedings of the Fifth International Workshop on New Worlds in Astroparticle Physics (Faro 2005), World Scientific (to be published).

[3] E. S. Seo et al., Astrophys. Journal 432, 656 (1994).

[4] J. Casaus, Cosmic-Ray Astrophysics with AMS-02, in Proceedings of the 28th International Cosmic Ray Conference (Tsukuba 2003), Universal Academy Press, Tokyo 2003. 